\title{
Successful electrical pacing for complete heart block complicating diphtheritic myocarditis ${ }^{1}$
}

\author{
Rodney E. Matisonn, Abdul S. Mitha, and Elliot Chesler \\ From the Cardiac Unit, Wentworth Hospital, and the University of Natal, Durban, South Africa
}

A case of severe diphtheria complicated by myocarditis and neurorespiratory paralysis is reforted. The myocarditis manifested with severe conduction disturbances including left bundle-branch block and highgrade second degree atrioventricular block leading to Adams-Stokes attacks. Temporary transvenous electrical pacing for 3 days was successful in the management of this complication, but positive pressure ventilation was later required for respiratory paralysis. This case illustrates the potential value of electrical pacing in diphtheritic myocarditis. Sporadic cases of diphtheria still occur and the case fatality ratio remains at 10 per cent, much of which is related to the occurrence of myocarditis.

As a result of widespread immunization the incidence of diphtheria has declined dramatically during the last half century. However, even in countries with effective public health programmes

'Supported by a grant from the Medical Research Council of South Africa. outbreaks still occur and it is noteworthy that the case fatality ratio remains constant at 10 per cent (Munford et al., 1974). Respiratory paralysis and myocarditis account for the majority of fatalities. The use of positive pressure ventilation has been a significant therapeutic advance but the potential

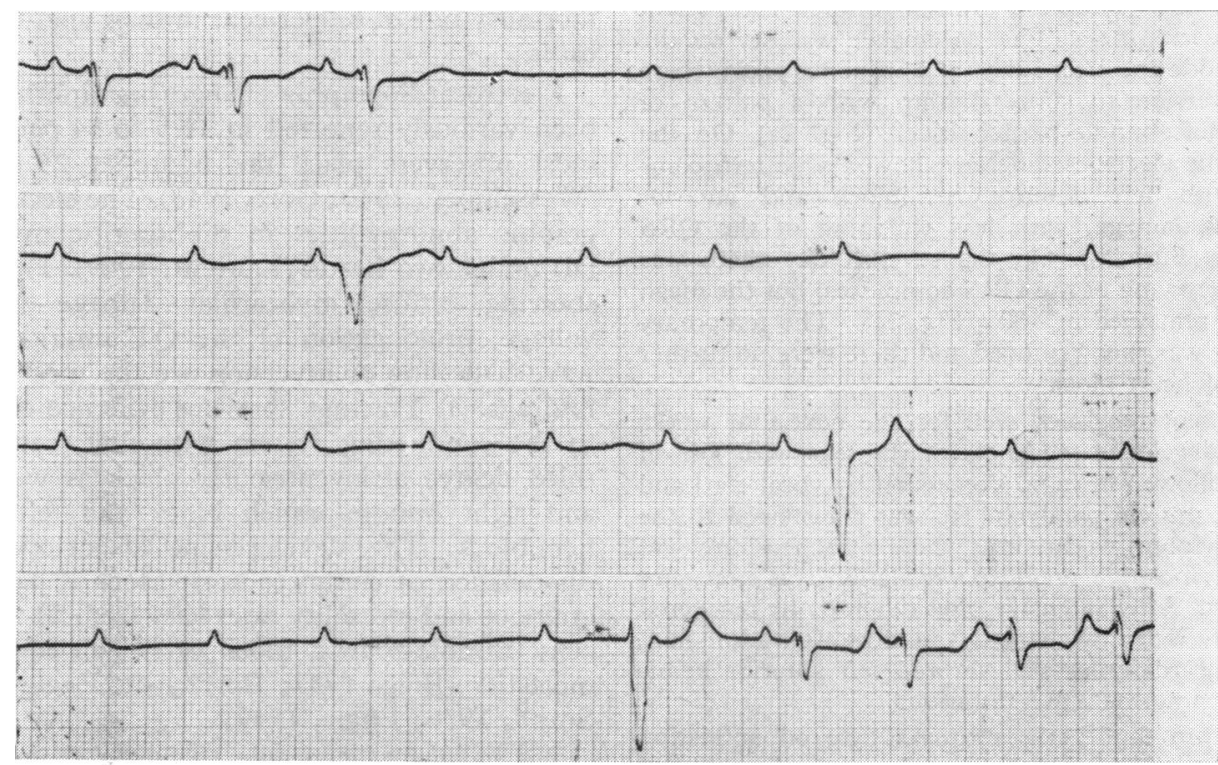

FIG. 1 Continuous electrocardiographic monitor strip on admission showing prolonged period of asystole. 
value of electrical pacing has not been exploited. J. F. Goodwin (1974, personal communication) was not aware of any report on the use of electrical pacing in diphtheritic myocarditis. In this paper we report the successful use of this technique in the management of a case of severe diphtheria complicated by complete heart block and respiratory paralysis.

\section{Case report}

An 8-year-old black boy was referred from an outlying hospital where he presented with a sore throat associated with cervical lymphadenitis. The possibility of diphtheria was entertained, but because a throat swab was negative for mycobacterium diphtheria, antidiphtheritic serum was unfortunately not given. $\mathrm{He}$ was treated with intramuscular penicillin, with some improvement. On the tenth day, however, he had a generalized seizure from which he recovered without neurological deficit. It was then noticed that there were episodes of bradycardia and asystole and he was transferred to our hospital.

On admission he was semiconscious and in a state of shock, with cold extremities. The pulse rate was 70 beats a minute with periods of asystole and the blood pressure was $50 / 30 \mathrm{mmHg}(6 \cdot 7 / 4 \cdot 0 \mathrm{kPa})$. The electrocardiogram showed high grade second degree atrioventricular block, with complete failure of atrioventricular conduction for periods of up to 8 seconds (Fig. 1). An intravenous infusion of isoprenaline restored sinus rhythm but there was a pattern of complete left bundlebranch block. A temporary pacing electrode was inserted via a superficial vein in the right antecubital fossa. A paced rate of 100 beats a minute restored the patient to full consciousness. The pacemaker was set on the demand mode and after 3 days of continuous pacing there was a reversion to sinus rhythm with a pattern of complete left bundle-branch block (Fig. 2a). On the fifth day the electrocardiogram showed a pattern compatible with left anterior hemiblock and anterior myocardial damage, with less widening of the QRS complexes (Fig. 2b). On the seventh day there was further narrowing of the $Q R S$ complexes, but the mean QRS axis remained at $-60^{\circ}$ (Fig. 2c). The temporary pacemaker was then removed and there were no further episodes of heart block.

The patient remained well for the next four weeks but then developed weakness of the legs with loss of reflexes, followed in rapid succession by vocal cord and intercostal muscle paralysis. He was transferred to the respiratory intensive care unit where a tracheostomy was performed; positive pressure ventilation was necessary for 28 days. After intensive physiotherapy he recovered completely. When discharged 3 months after admission, the electrocardiogram still showed left anterior hemiblock with a normal QRS duration.

The serum diphtheria antitoxin titre on admission was 25 units $/ \mathrm{ml}$, which, in an unimmunized individual who had not received antidiphtheritic serum, is regarded as diagnostic of diphtheria.

\section{Comment}

Although diphtheria is a preventable disease, sporadic cases still occur. For example, a recent analysis of diphtheria deaths in the United States for the period 1959 to 1970 (Munford et al., 1974) has confirmed the overall dramatic decrease in the reported morbidity and mortality but clearly indicates that the case fatality ratio remains at 10 per cent. Several factors are concerned in the risk of dying from diphtheria. Firstly, survival is more common in patients who receive antitoxin early in the course of the disease. Secondly, strain-virulence and host factors are involved and case fatality ratios are highest in the extreme age groups, with children under the age of 5 years being particularly vulnerable. Persons who have received recommended doses of toxoid are at less risk than those who have had none. However, approximately 10 per cent of cases of diphtheria occur in persons who have been fully immunized, and the risk of mortality appears to be higher in small outbreaks, suggesting that delayed recognition with late administration of appropriate therapy is operative. It seems, therefore, that the potential for confrontation with diphtheria is always present and that unless appropriate therapeutic measures are adopted fatalities are unavoidable.

The most lethal complication of the disease is myocarditis which is a result of circulating exotoxin. Myocarditis occurs in approximately 10 per cent of cases, and Hoyne and Welford (1934) found a mortality rate of 62 per cent in their analysis of 496 cases.

Electrocardiographic changes in diphtheria have been variously reported in 16.5 to 84 per cent but most observers place the figure at 25 per cent (Wesselhoeft, 1940; Gore, 1948). The electrocardiographic abnormalities in diphtheritic myocarditis fall under two heads (Laurent, 1949). 1) Tracings showing diffuse myocardial damage with low voltage, prolongation of the QT interval, and T wave flattening or inversion are the most frequent findings. 2) Tracings showing evidence of damage to the conducting system with first, second, and third degree atrioventricular block as well as left and right bundle-branch block and left anterior hemiblock. The development of atrioventricular dissociation or complete heart block is particularly ominous and mortality rates vary from 54 to 100 per cent. Bundle-branch block carries an equally high mortality (Bigg, 1937; Burkhardt, Eggleston, and Smith, 1938; Engle, 1949).

We have not been able to find a report of successful electrical pacing in this disease. Our case suggests, however, that the chance of survival may 


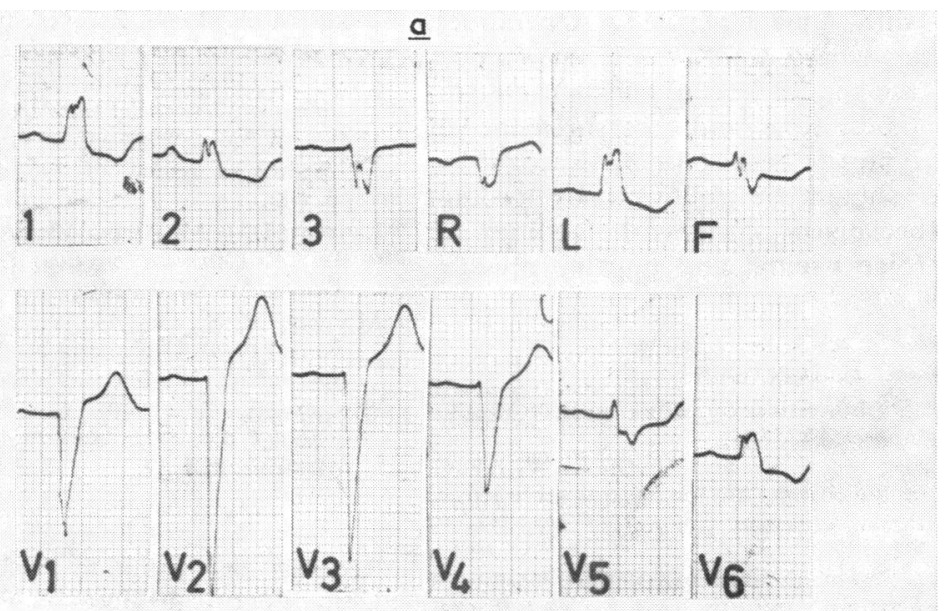

$\underline{\text { b }}$
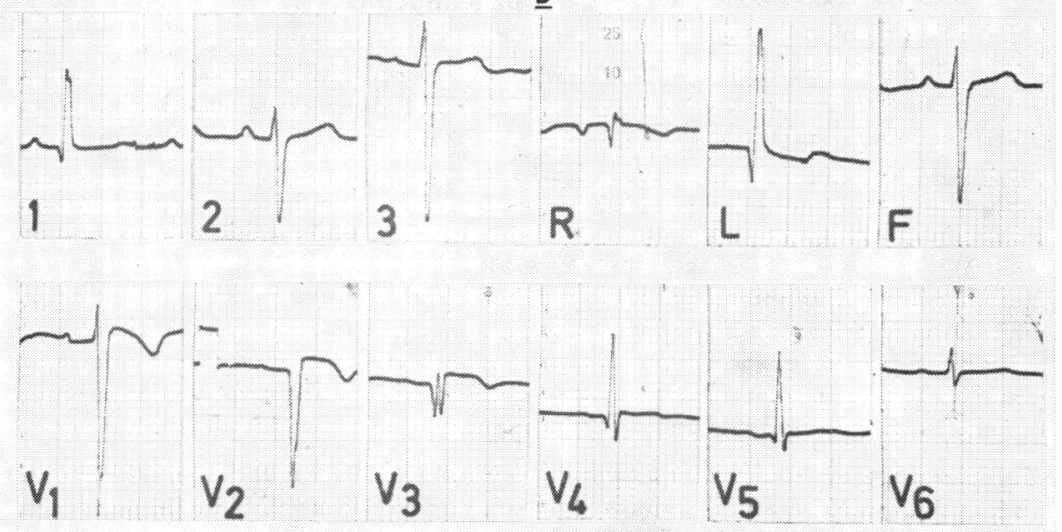

$\underline{\mathrm{C}}$
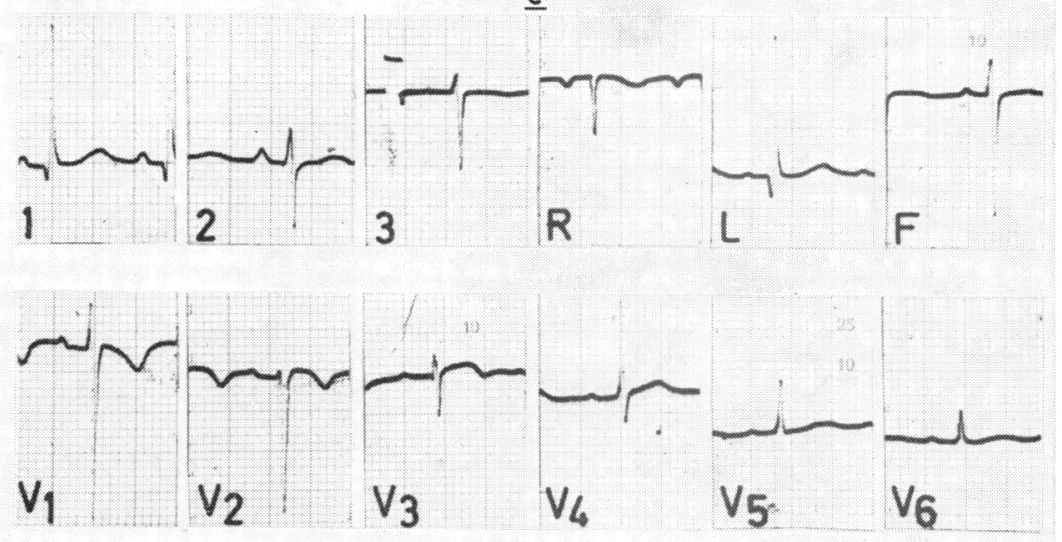

FIG. 2 a) Electrocardiogram immediately after cessation of 3 days of continuous pacing showing sinus rhythm with complete left bundle-branch block. b) Electrocardiogram 5 days after cessation of pacing showing left anterior hemiblock and anterior myocardial damage. c) Electrocardiogram 7 days after cessation of pacing showing left anterior hemiblock and narrowing of the QRS complexes compared with a) and b) above. 
be improved with modern pacing techniques. Even in countries where diphtheria is uncommon, diagnosis of a case should lead to constant electrocardiographic monitoring and measurement of intracardiac enzymes (Chesler, 1958). Recognition of myocarditis should be followed by prompt referral to a specialized centre with facilities for monitoring, cardiac pacing, and positive pressure ventilation.

\section{References}

Begg, N. D. (1937). Diphtheritic myocarditis : an electrocardiographic study. Lancet, 232, 857.

Burkhardt, E. A., Eggleston, C., and Smith, L. W. (1938). Electrocardiographic changes and peripheral nerve palsies in toxic diphtheria. American fournal of the Medical Sciences, 195, 301.

Chesler, E. (1958). Serum glutamic oxalacetic transaminase levels in diphtheritic myocarditis. British Heart fournal, $20,244$.
Engle, M. A. (1949). Recovery from complete heart block in diphtheria. Pediatrics, 3, 222.

Gore, I. (1948). Myocardial changes in fatal diphtheria. American fournal of the Medical Sciences, 215, 257.

Hoyne, A., and Welford, N. T. (1934). Diphtheritic myocarditis, a review of 296 cases. Fournal of Pediatrics, $5,642$.

Laurent, L. J. M. (1949). Electrocardiography in diphtheria. In The Common Infectious Diseases, p. 41. Ed. by H. S. Banks. Arnold, London.

Munford, R. S., Ory, H. W., Brooks, G. F., and Feldman, R. A. (1974). Diphtheria deaths in the U.S.A. 1959-1970. Fournal of the American Medical Association, 229, 1890.

Wesselhoeft, G. (1940). Communicable diseases: cardiovascular disease in diphtheria. New England fournal of Medicine, 223, 57.

Requests for reprints to Professor E. Chesler, Cardiac Unit, Wentworth Hospital, P. B. Jacobs, Natal 4026, South Africa. 